\title{
Esterified Anabolic Androgen-Induced Liver Injury in a Hepatitis C Virus-Positive Patient: A Case Report
}

Kyrillus S. Shohdy, ${ }^{1}$ Rasmia M. El Gohary. ${ }^{2}$

\begin{abstract}
Background: Cases of drug induced liver injury still perplex gastroenterologists due to its wide range of presentations that mimic acute and chronic liver conditions. Moreover, matters get complicated when clinicians face the possibility of drug-induced injury in the presence of pre-existing chronic liver disease. Case: A 69 year-old male who was recently discovered to have a hepatitis C viral infection presented with acute manifestations (mixed cholangio-hepatocellular injury) not fully explained by the underlying chronic disease, we suspected an idiosyncratic reaction from an esterified anabolic androgen. His manifestations have appeared acutely after the drug intake and include acute onset of jaundice, abdominal pain, pruritus and choluria. He was improving on drug discontinuity and conservative measures during his brief hospital stay. Conclusion: The underlying chronic disease constitutes a dilemma in diagnosis of superimposed drug-induced liver injury, as the proof of causality is a daunting task. In such cases, it is tempting to link such new emerging manifestations to be a flare-up of the underlying chronic disease rather than to the drug. However, certain clues helped to point this clinical presentation towards a drug-induced liver injury.
\end{abstract}

Keywords:Drug-Induced Liver Injury; Hepatitis; Steroids; Biological Markers (Source: MeSH, NLM).

About the Author: Kyrillus is currently pursuing a rotating internship year at Kasr Al Ainy School of Medicine, Cairo, Egypt af ter graduating from the 6-year program. He is interested in research realms where our understanding of the biological proces ses behind certain pathology enables us to design an effective and targeted therapy.

\section{Introduction}

Cases of drug induced liver injury (DILI) still perplex gastroenterologists due to its wide range of presentations that mimic acute and chronic liver conditions. The liver is the most common organ prone to toxicity. However, the absence of internationally accepted diagnostic criteria for DILI along with underreporting and underrecognition make the overall incidence variable which ranges from 1 in 10,000 to 1 in 100,000 patients. ${ }^{1,2}$ For instance, in one population-based cohort study, the incidence was 14 cases in $100,000 .{ }^{3}$ It is worth noting that hepatotoxicity was found to be the most common cause behind drug withdrawal or drug usage modification. ${ }^{2}$

Suspicion for DILI with a rarely reported substance may be unlikely in the presence of an underlying chronic liver disease such as hepatitis $\mathrm{C}$ virus (HCV) infection. Furthermore, studies that assess the effect of HCV on the progression of DILI in cases of non-acetaminophen toxicity like anabolic steroids are limited in number. The important risk factors for the progression of DILI are age ( $>55$ years old), pregnancy and chronic alcohol consumption, ${ }^{4}$ meanwhile diabetes mellitus is a controversial risk factor., ${ }^{5,6}$

\section{Key Points: \\ - The clinical and biochemical presentation of drug-induced liver injury mimics a broad array of liver diseases. \\ - In some instances, liver biopsy can provide unequivocal differentia- tion between flare-ups of chronic liver diseases and superimposed liver damage caused by medications. \\ - Short timespan between drug intake and the onset of liver injury manifestations, the pattern of hepatotoxicity and presence of conju- gated hyperbilirubinemia could point to the diagnosis of drug-induced liver injury. \\ - Liver function tests should be performed before starting anabolic steroids, either oral or injectable, in endemic areas of asymptomatic chronic liver diseases.}

The naturally occurring testosterone hormone can be modified for therapeutic use by one of two ways: esterification or alkylation of the 17a-hydroxyl group of the testosterone molecule (Figure 1). The alkylated forms can be taken orally and are considered a common cause of liver injury, ${ }^{7}$ whereas the esterified forms such as testosterone enanthate (TE) are considered debatable cause for DILI as these drugs are taken illicitly and case reports are the only available evidence. ${ }^{7.8}$ This case may open

Figure 1. Structure of testosterone (A) and its esterified form, testosterone enanthate (B)

A

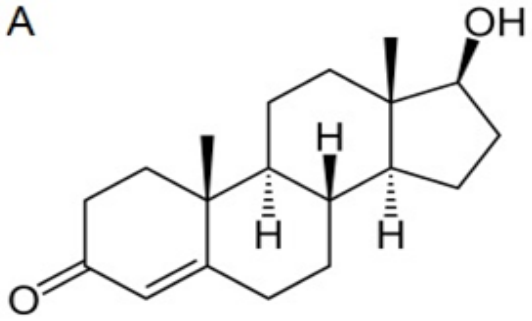

B<smiles>CCCCCCC(C)O[C@H]1CC[C@H]2[C@@H]3CCC4=CC(=O)CC[C@]4(C)[C@H]3CC[C@]12C</smiles>

Submission: Jul 6, 2015

Acceptance: Sep 22, 2015

Publication: Oct 20, 2015

Process: Peer-reviewed

${ }^{1}$ Faculty of Medicine, Cairo University, Cairo, Egypt.

${ }^{2}$ Internal Medicine Department, Faculty of Medicine, Cairo University, Cairo, Egypt.

\section{Correspondence:}

Kyrillus S. Shohdy

Address: Faculty of Medicine, Cairo University, Cairo, Egypt.

Email: kerosam501@gmail.com 
Table 1. Case Summary.

\begin{tabular}{|c|c|}
\hline Duration of culprit drug therapy & 7 days \\
\hline Time to onset of symptoms & 8 days \\
\hline Clinical presentation & $\begin{array}{l}\text { Jaundice, abdominal pain, pruritus, } \\
\text { choluria }\end{array}$ \\
\hline Time to admission & 21 days \\
\hline Initial $\mathrm{R}$ ratio & 3.3 (Mixed pattern) \\
\hline $\begin{array}{l}\text { Time to first detection of lab } \\
\text { abnormalities }\end{array}$ & 21 days \\
\hline $\begin{array}{l}\text { Liver function tests at time of } \\
\text { admission }\end{array}$ & $\begin{array}{l}\text { ALT }=6.9 \text { times ULN* } \text {, AST= } 5.9 \text { times } \\
\text { ULN, ALK P=2.25 times ULN. total bili- } \\
\text { rubin } 30.0 \mathrm{mg} / \mathrm{dL} \text {, direct } 28.0 \mathrm{mg} / \mathrm{dL} \text {. }\end{array}$ \\
\hline After 5 days of admission & $\begin{array}{l}\text { ALT, AST and Alk P showed mild decli- } \\
\text { ne, total bilirubin } 27.0 \mathrm{mg} / \mathrm{dL} \text {, direct } \\
25.0 \mathrm{mg} / \mathrm{dL} \text {. }\end{array}$ \\
\hline Time to resolution & Not applicable \\
\hline
\end{tabular}

Lengend: ULN= the upper limit of normal.

the gate for larger scale studies on anabolic steroids to investigate the possibility that HCV may be an important risk factor for the development of idiosyncratic reactions, those which are unpredictable and not dose-dependent, as it is with acetaminophen. ${ }^{9}$

One important measure to define the pattern of hepatotoxicity is the $R$ value which is equal to serum (ALT/ALT upper limit of normal $) \div($ Alk $P /$ Alk $P$ upper limit of normal). This classification was proposed by Zimmerman and later adopted by the Council for International Organisation of Medical Sciences (CIOMS), where the patterns of acute liver injury are determined as follows i) Hepatocellular: $R$ value $\geq 5$ ii) Mixed hepatitic/cholestatic: $R$ value between 2-5 iii) Cholestatic pattern $R$ value $\leq 2 .{ }^{4,10}$ Our case is an example of the mixed pattern. A major limitation facing this classification is that elevations in biochemical parameters, such as the ALT, can represent an adaptive response to the drugs and may not point out a true injury. ${ }^{11}$

\section{The Case}

A 69 year-old Egyptian male patient presented to our emergency room with an acute onset of jaundice, abdominal swelling and itching. Three weeks before presenting to our emergency room, he sought medical advice for his erectile dysfunction issue. He has a long history of diabetes and hypertension which were controlled with glimepiride $3 \mathrm{mg}$ and bisoprolol $5 \mathrm{mg}$ respectively. His andrologist prescribed tadalafil $5 \mathrm{mg}$ and testosterone enanthate $250 \mathrm{mg}$, the latter, which was prescribed for unclear reason, was given twice intramuscularly with a one-week interval. The first injection caused an epigastric pain described by the patient as a burning sensation with slight abdominal tenderness, but it was after the second injection that caused his condition to worsen with an acute onset of jaundice, abdominal swelling, itching and dark colored urine and pale stools. These symptoms have continued to the time of admission to our emergency room. on physical examination he was afebrile, the pulse was $55 \mathrm{bpm}$ and his blood pressure was 135/75 $\mathrm{mmHg}$. The jaundice was strikingly apparent in his entire skin and mucous membranes, and the head and neck examination revealed bilateral parotid enlargement. The patient denied alcohol consumption or any herbal or dietary supplements.

Abdominal examination revealed the presence of generalized abdominal distention, epigastric tenderness, enlargement of the liver with a sharp border and shifting dullness revealed ascites. His past medical history is unremarkable for previous surgeries or chronic morbidities except for an attack of myocardial infarction. His liver function tests (LFTs) showed a mixed hepatitis/cholestasis pattern where alanine transaminase (ALT) was $240 \mathrm{U} / \mathrm{L}$ [6.9 times the upper limit of normal], aspartate transaminase (AST) $264 \mathrm{U} / \mathrm{L}[5.9$ times the upper limit of normal], alkaline phosphatase (Alk P) $270 \mathrm{U} / \mathrm{L}$ (2.25 times the upper limit of normal), hence the calculated $R$ value is 3.3 , total bilirubin $30.0 \mathrm{mg} / \mathrm{dL}$, (direct $28.0 \mathrm{mg} / \mathrm{dL}$ ), albumin $3.7 \mathrm{~g} / \mathrm{dL}$ and eosinophils $2 \%$. The abdominal ultrasound revealed hepatomegaly with no dilatation of the intrahepatic biliary tract radicles with normal sized kidneys and his autoimmune and virology screening was negative except that his HCV RNA was qualitatively positive. The patient was not aware of being HCV positive before. We restricted our screening to the recommendations of the American College of Gastroenterology clinical guidelines for investigating a case of suspected DILI that included anti-nuclear antibody, anti-smooth muscle antibody, IgG level, Anti-HAV IgM, HBSAg, anti-HBC IgM, anti-HCV and HCV RNA. ${ }^{10}$ On follow-up, after 5 days of admission, the total and direct bilirubin were 27

Table 2. RUCAM criteria to prove causality in DILI cases.

$\begin{aligned} & \text { Criteria } \\ & \text { Time between drug start and liver enzyme }\end{aligned}$
$\begin{aligned} & \text { Possible Scores } \\ & \text { elevations }\end{aligned}$


$\mathrm{mg} / \mathrm{dl}$ it $25 \mathrm{mg} / \mathrm{dl}$ respectively and LFTs were ALT $186 \mathrm{U} / \mathrm{L}$, AST 203 U/L, Alk P 189 U/L.

The symptoms of the patient were improving slowly on withdrawal of TE. Simultaneously, intravenous fluids and $\mathrm{N}$-acetylcysteine were administered while monitoring for the development of coagulopathy or encephalopathy. There were no clinical benefits found in the literature regarding the use of ursodeoxycholic acid (UDCA) in the mixed hepatotoxicity pattern except for lowering alkaline phosphatase levels. ${ }^{12}$ The patient left against medical advice so a follow-up could not be reported. Written informed consent was obtained from the patient for publication of this case report.

\section{Discussion}

The clinical and biochemical presentation of drug-induced liver injury mimics a broad array of liver diseases. However, the most common clinical presentation resembles hepatocellular jaundice or cholestatic liver disease. ${ }^{13}$ According to the LiverTox $®$ (Available from: http://livertox.nih.gov/AndrogenicSteroids.htm\#top, updated 2015 June 23; cited 2015 July 21) toxicity with injectable anabolic steroids (esterified testosterone) is not well documented. In the review of the literature, we found two cases that developed liver toxicity (acute cholestasis) while using esterified testosterone, ${ }^{14,15}$ and one case that developed a hepatic adenoma. ${ }^{16}$ Moreover, preclinical studies showed that long term use of esterified testosterone like testosterone enanthate can cause an increase in liver enzymes and a decrease in HDL-cholesterol. ${ }^{17}$ On the other hand, one study by Marquardt et al in 1964 showed failure of esterified anabolic steroids (non C 17-alkylated) to produce abnormal liver function tests. ${ }^{8}$ Further studies are needed to assess the short and long term effects of esterified testosterones on the liver.

The differential diagnoses of this case includes acute viral hepatitis, autoimmune hepatitis and other less common viruses e.g. cytomegalovirus and Epstein-Barr virus. Acute hepatitis C can masquerade as DILI in $1.3 \%$ of cases especially with acute hepatocellular injury and suggestive HCV RNA testing. ${ }^{\circ}$ However, these two findings do not apply to our case, for example, Chalasani et al. recruited 300 cases with suspected DILI in a prospective study and found that DILI is an unlikely diagnosis with only 9 positive cases $(3 \%)$ and in 4 of them the final diagnosis turned out to be an $\mathrm{HCV}$ infection. ${ }^{5}$ Another study, recruited 570 cases, has excluded 59 cases for an alternative cause of injury which was viral hepatitis on 11 occasions. A total of $446(78 \%)$ cases were deemed to be idiosyncratic hepatotoxicity. ${ }^{6}$ Although viral hepatitis is a valid differential diagnosis in suspected DILI cases, studies have shown that it is a less likely cause of injury in idiosyncratic liver injury.

The existence of underlying liver disease can still hinder a straightforward diagnosis of DILI in general, since the flare-up of the chronic disease caused by viral hepatitis cannot be ruled out completely. In some instances, liver biopsy can provide unequivocal differentiation between flare-ups and superimposed damage by the offending drug. Other important clues are the short time period between the culprit drug intake and the onset of clinical manifestations, the pattern of hepatotoxicity and presence of conjugated hyperbilirubinemia. ${ }^{1,13}$ We depended on these previous three clues for considering DILI as a possibility in this case. We have also implemented the Roussel Uclaf Causality Assessment Method (RUCAM), which was developed in 1993 by a group of international experts; ${ }^{4}$ our case achieved a possible $(+5)$ score (refer to Table 2 ). An interesting review from the Spanish Group for the Study of Drug-Induced Liver Disease points out the strengths and weaknesses of the available causality assessment methods. ${ }^{18}$

The prevention of idiosyncratic drug reactions is a daunting task; however the future is promising with progress towards the development of biomarkers, where the genetic profile of every person can be screened to determine whether a certain drug can cause an idiosyncratic toxicity. This advancement will be very important to those with chronic liver disease, for example, Abacavir which is an antiviral used in human immunodeficiency virus (HIV) treatment, can cause severe hypersensitivity reactions and hepatotoxicity. These reactions have been found to occur in patients with human leukocyte antigen- $B^{*} 5701$ and genetic screening prior to Abacavir intake is strongly advised by the Food and Drug and Administration (FDA) to prevent such severe reactions. ${ }^{19}$ In addition, the HLA-B* 5701 genotype has been linked with flucloxacillin induced liver injury. ${ }^{20}$ Though these genetic linkage studies can predict what was thought to be unpredictable idiosyncrasies, there is still a long way to reach widely accepted predictive biomarkers.

\section{Conclusion}

Two clinical notes learnt from this case; first, LFTs are recommended before starting anabolic steroids either oral or injectable in endemic areas with asymptomatic chronic liver diseases such as HCV. Second, in any case of acute liver injury within endemic areas, stigmata of chronic liver disease have to be investigated thoroughly by clinical, biochemical and radiological means. Physicians have to take into consideration that HCV as well as other chronic diseases e.g. alcoholic liver disease and nonalcoholic fatty liver disease (NAFLD) leave the liver susceptible to steroid toxicity. In contrast to alcoholic hepatitis and HBV, HCV patients do not experience icteric flares, which along with the history of hepatotoxic agent intake, latency to DILI onset, and biochemical and histological features at presentation raise the index of suspicion to DILI. However, due to diagnostic limitations it is difficult to distinguish with a high-degree of certainty between a spontaneous disease flare-up and a DILI episode. 


\section{Case Report}

\section{References}

1. Navarro VJ, Senior JR. Drug-related hepatotoxicity. N Engl J Med. 2006 Feb 16;354(7):731-9.

2. Devarbhavi H. An update on drug-induced liver injury. J Clin Exp Hepatol. 2012 Sep; 2(3): 247-59.

3. Sgro C, Clinard F, Ouazir K, Chanay H, Allard C, Guilleminet C, et al. Incidence of drug-induced hepatic injuries: a French population-based study. Hepatology. 2002 Aug;36(2):451-5.

4. Danan G, Benichou C. Causality assessment of adverse reactions to drugs-I. A novel method based on the conclusions of international consensus meetings: application to drug-induced liver injuries. J Clin Epidemiol. 1993 Nov;46(11):1323-30.

5. Chalasani N, Fontana RJ, Bonkovsky HL, Watkins PB, Davern T, Serrano J, et al. Causes, clinical features, and outcomes from a prospective study of drug-induced liver injury in the United States. Gastroenterology. 2008 Dec;135(6):1924-34, 1934.e1-4.

6. Andrade RJ, Lucena MI, Fernández MC, Pelaez G, Pachkoria K, García-Ruiz $\mathrm{E}$, et al. Drug-induced liver injury: an analysis of 461 incidences submitted to the Spanish registry over a 10-year period. Gastroenterology. 2005 Aug;129(2):512-21.

7. Synder P. Androgens. In: Brunton L, Chabner B, Knollman B, editors. Goodman at Gilman's the pharmacological basis of therapeutics. 12th ed. New York: McGraw-Hill; 2011. p. 1195-208.

8. Marquardt GH, Logan CE, Tomhave WG, Dowben RM. Failure of non-17alkylated anabolic steroids to produce abnormal liver function tests. J Clin Endocrinol Metab. 1964 Dec;24:1334-6.

9. Nguyen GC, Sam J, Thuluvath PJ. Hepatitis C is a predictor of acute liver injury among hospitalizations for acetaminophen overdose in the United States: a nationwide analysis. Hepatology. 2008 0ct;48(4):1336-41.

10. Chalasani NP, Hayashi PH, Bonkovsky HL, Navarro VJ, Lee WM, Fontana Rj; Practice Parameters Committee of the American College of Gastroenterology. ACG Clinical Guideline: the diagnosis and management of idiosyncratic drug-induced liver injury. Am J Gastroenterol. 2014 Jul;109(7):950-66; quiz 967.
11. Watkins PB, Seeff LB. Drug-induced liver injury: summary of a single topic clinical research conference. Hepatology. $2006 \mathrm{Mar} ; 43(3): 618-31$.

12. Huang Y-S. S1881 the therapeutic efficacy of ursodeoxycholic acid (UDCA) in drug-induced liver injury: results of a randomized controlled trial. Gastroenterology. 2010 May;138(5):S-809.

13. Kaplowitz N. Drug-induced liver disease. 3rd ed. Philadelphia: Elsevier Inc; 2013. p. 3-14.

14. Yoshida EM, Erb SR, Scudamore CH, Owen DA. Severe cholestasis and jaundice secondary to an esterified testosterone, a non- $\mathrm{C} 17$ alkylated anabolic steroid. J Clin Gastroenterol. 1994 Apr;18(3):268-70.

15. Savvidou S, Gagalis A, Efstratiou I, Kalambakas A. Anabolic androgen-induced intrahepatic cholestasis presented with normal $\gamma$-glutamyl-transpeptidase. J Interdiscipl Histopathol. 2014;2(2):98-103.

16. Carrasco D, Prieto M, Pallardó L, Moll JL, Cruz JM, Muñoz C, et al. Multiple hepatic adenomas after long-term therapy with testosterone enanthate. Review of the literature. J Hepatol. 1985;1(6):573-8.

17. Tyagi A, Rajalakshmi M, Jeyaraj DA, Sharma RS, Bajaj JS. Effects of longterm use of testosterone enanthate. II. Effects on lipids, high and low density lipoprotein cholesterol and liver function parameters. Int J Androl. 1999 Dec;22(6):347-55.

18. García-Cortés M, Stephens C, Lucena MI, Fernández-Castañer A, Andrade RJ; Spanish Group for the Study of Drug-Induced Liver Disease (Grupo de Estudio para las Hepatopatías Asociadas a Medicamentos GEHAM). Causality assessment methods in drug induced liver injury: strengths and weaknesses. J Hepatol. 2011 Sep;55(3):683-91.

19. Liss G, Rattan S, Lewis JH. Predicting and preventing acute drug-induced liver injury: what's new in 2010? Expert Opin Drug Metab Toxicol. 2010 Sep;6(9):1047-61.

20. Daly AK, Donaldson PT, Bhatnagar P, Shen Y, Pe'er I, Floratos A, et al. $H L A-B^{\star} 5701$ genotype is a major determinant of drug-induced liver injury due to flucloxacillin. Nat Genet. 2009 Jul;41(7):816-9.

\section{Acknowledgments}

The authors would like to thank Prof. Hala El Gendy, MD for her assistance in the management of the case.

Conflict of Interest Statement a Funding

The author has no funding, financial relationships or conflicts of interest to disclose.

\section{Author Contributions}

Conception and design the work/idea, Write the manuscript, Contribution of patients or study material: KSS, REG. Collect data/obtaining results, Analysis and interpretation of data, Write the manuscript: KSS. Contribution of patients or study material, Administrative or technical advice: REG.

Cite as:

Shohdy KS, El Gohary RM. Esterified Anabolic Androgen-Induced Liver Injury in a Hepatitis C Virus-Positive Patient: A Case Report. Int J Med Students. 2015 Sep-Dec;3(3):151-4. 